\title{
Determinación de criterios generales para el diseño de juegos serios: modelo metodológico integrador
}

\author{
Laura M. Londoño ${ }^{1^{*}}$ y Miguel D. Rojas ${ }^{2}$ \\ (1) Facultad de Ingeniería, Departamento de Ingeniería Industrial, Universidad de Antioquia, Calle 70 No. 52 - 21, Medellín- \\ Colombia (correo-e: laura.Iondono9@udea.edu.co). \\ (2) Facultad de Minas, Departamento de Ingeniería de la Organización, Universidad Nacional de Colombia, Carrera 80 \\ no.65-223, Medellín-Colombia (correo-e: mdrojas@unal.edu.co).
}

* Autor a quien debe ser dirigida la correspondencia.

Recibido Jul. 30, 2020; Aceptado Sep. 28, 2020; Versión final Nov. 16, 2020, Publicado Feb. 2021

\begin{abstract}
Resumen
El objetivo de esta investigación es identificar criterios generales para el diseño de juegos serios y plantear un modelo metodológico integrador, amigable y versátil que facilite las actividades de futuros diseñadores de juegos. Se realizó una revisión bibliográfica de modelos y metodologías para el diseño de juegos serios propuestos en los últimos 20 años. Los hallazgos se clasificaron en 8 categorías generales con las cuales se propone el modelo metodológico integrador. El modelo cuenta con tres fases: pre-diseño, diseño y testeo. Cada fase tiene actividades específicas para el diseño del instrumento e integra los criterios encontrados: establecer objetivos, caracterizar público objetivo, estudiar la literatura, incorporar elementos de juego, realizar prototipos y probar el juego. Se concluye que el modelo metodológico propuesto es una herramienta novedosa, sencilla e intuitiva, que puede ser utilizada en el diseño de juegos serios de manera presencial o en ambientes virtuales.
\end{abstract}

\section{General criteria determination for designing serious games: integrative methodological model}

\begin{abstract}
The objective of this research study is to identify general criteria for designing serious games to develop an integrative methodological model that is user-friendly, versatile, and that facilitates game design. A literature review of serious game design models and methodologies in the last 20 years is performed. The results were classified into eight general categories that are used to propose an integrative methodological game design model. The model has three phases: pre-design, design, and testing. Each phase has specific activities for instrument design and integrates the criteria found in the literature: establishing objectives, target audience characterization, studying the literature, incorporating game elements, prototyping, and game testing. It is concluded that the proposed methodological model is a novel tool, simple, and intuitive that can be used to design serious games in person or in virtual environments.
\end{abstract}

Keywords: serious games; development; design methodology; model; education 


\section{INTRODUCCIÓN}

En las últimas décadas los juegos han sido incluidos de forma creciente en ambientes educativos y son cada día más difundidos para lograr procesos efectivos de enseña-aprendizaje, en los cuales los participantes aprenden por medio de aplicación de conocimientos, toma de decisiones, práctica y simulación de escenarios. Para Mogrovejo, Mamani, y Tipo (2019) el poder de la simulación y del juego reside en la realidad de la práctica comunicativa en la que están envueltos los estudiantes, del análisis de la situación enfrentada y de la toma de decisiones. No obstante, son numerosos los términos con los cuales se designa a los juegos dentro de contextos formativos, siendo el término juegos serios el de mayor aceptación y desarrollo dentro de la literatura. Para Djaouti, Alvarez, Jessel, y Rampnoux (2011) el término "serious games", traducido como juegos serios, fue usado y definido por primera vez por el autor Clark Abt en 1970; quien era un investigador estadounidense durante la guerra fría y tenía como objetivo el uso de juegos para entrenar y educar, de ésta manera, Abt definió los juegos serios como: "juegos que tienen un explícito y cuidadosamente pensado propósito educativo y no están destinados a ser jugados principalmente por diversión; lo cual no significa que los juegos serios no sean, o no deban ser entretenidos"; desde entonces son numerosas las definiciones y derivaciones que surgen con respecto a los juegos serios y en general, juegos diseñados con propósito principalmente educativo. Para la investigación se tomará el término juegos educativos (Prieto et al., 2017) como sinónimo de juego serios, dado que son términos comúnmente asociados.

Los juegos serios según la definición más simple son "juegos que no tienen al entretenimiento, el disfrute y la diversión como propósito principal" (Michael y Chen, 2006), además, los autores proponen que éstos juegos utilizan el medio artístico para transmitir un mensaje, enseñar una lección o proveer una experiencia, sin embargo, Breuer y Bente (2010) consideran que igualmente, existen juegos serios que no tienen un enfoque educativo directo, pero que a pesar de esto, son considerados serios, es decir, son juegos en los cuales el propósito educativo no es evidente, sin embargo, logran educar en aspectos específicos. Adicionalmente, son considerados como "un concurso mental jugado con una computadora, de acuerdo con reglas específicas y que utiliza entretenimiento para promover entrenamiento gubernamental o corporativo, educación, salud, políticas públicas y objetivos de comunicación estratégica“ (Zyda, 2005), definición en la cual los juegos serios son limitados a interacciones por medio de computadora; de esta manera, del anterior conjunto de definiciones es posible evidenciar diversas propuestas que surgen en torno a juegos serios y que difieren en algunos elementos, pero que, sin embargo, guardan una relación en su característica principal y es que éstos juegos son utilizados para más que diversión, dado que son particularmente útiles, ya que promueven la participación de estudiantes en el proceso de aprendizaje en comparación con métodos de enseñanza tradicionales (Silva, 2020).

Así, a medida que los juegos se han vuelto usuales dentro de los procesos educativos, se ha fundado la necesidad de crear herramientas efectivas y metodologías específicas para su diseño, desarrollo y entrega (Nadolski et al., 2008), es decir, metodologías para la concepción, elaboración y puesta a punto y aplicación final. Específicamente en el área de diseño y desarrollo de juegos serios, existen numerosos modelos que sirven como pauta para ser imitados o reproducidos y metodologías, que son conjuntos de métodos, que intentan guiar a los diseñadores en el proceso de definición, creación, validación y aplicación final de las herramientas, incluso se evidencian propuestas metodológicas que dan pautas para el diseño de juegos en áreas o situaciones específicas. Sin embargo, Silva (2020) plantea que no existe una única forma de desarrollar juegos educativos, ya que depende de factores como público objetivo, tema a enseñar y tipo de juego; para Yusoff, Crowder, Gilbert, y Wills (2009) existen normas y pautas poco claras, por lo cual es difícil establecer si un juego serio realmente cumple con los requisitos o expectativas del estudiante.

Para el diseño de juegos en general existen diversas propuestas, habitualmente centradas en la inclusión de diversión y entretenimiento como principales factores del proceso de diseño; sin embargo, la introducción de un objetivo principal de aprendizaje dentro de los juegos serios, hacen que el proceso específico para este tipo de propuestas no sea fácil, ya que puede involucrar diferentes profesiones como diseñadores, programadores y expertos en el tema a enseñar, haciendo complejo el proceso de comunicación entre estos (Silva, 2020) y, es común, que los juegos se basen en ideas de los diseñadores y desarrolladores, debido a que algunos conocimientos son difíciles de integrar y entonces, se mantiene el factor diversión en primer lugar (Barbosa et al, 2014). De esta manera, son numerosos los factores que influyen en la efectividad de un juego serio y en su exitosa aplicación al público objetivo. Para Mitgutsch y Alvarado (2012) no solo la mentalidad de los jugadores y el marco contextual influyen en el impacto de los juegos serios, sino también la manera en que el objetivo de dichos juegos se canaliza a través del diseño; además, Rooney (2012) plantea que un marco pedagógico sólido y la integración de elementos de juego que mantengan el compromiso del jugador, son elementos esenciales para la efectividad de los juegos serios. Otros investigadores creen que los juegos deben combinar apropiadamente diseño y pedagogía, para optimizar sus beneficios y efectividad en la educación (Ibrahim y Jaafar, 2009). Se evidencia entonces, diversidad de propuestas y factores a tener en 
cuenta en el diseño de juegos serios, haciendo difícil la tarea del diseñador, más aún sin un modelo metodológico que incluya la variedad de criterios a tener en cuenta.

Como una alternativa para enfrentar la falta de claridad, diversidad de metodologías y modelos existentes para el diseño de juegos serios y los diferentes factores que influyen en su diseño, la presente investigación se basa en la identificación de criterios generales a partir del análisis de otras metodologías, los cuales son resultado de una revisión bibliográfica realizada en la base de datos Scopus y complementada con documentos referentes encontrados por fuera de dicha base de datos, a partir de estos, se propone un modelo metodológico integrador para el diseño de juegos serios. El modelo metodológico propuesto consta de tres fases fundamentales: pre-diseño, diseño y testeo, las cuales a su vez tienen elementos específicos propios para diseño de herramientas, con lo cual se busca exponer un proceso íntegro, versátil e intuitivo que facilite a diseñadores, la tarea de integrar conceptos y temáticas educativas, con juegos.

\section{METODOLOGÍA}

Para el desarrollo de la investigación, inicialmente se realizó una revisión bibliográfica en la base de datos Scopus, con el objetivo de determinar las metodologías existentes para el diseño de juegos serios o juegos educativos. La revisión se fundamenta en la guía propuesta por Velásquez (2015) y para ella se utilizó el término juego, dado que incluye las posibles variantes como juegos serios, juegos educativos, aprendizaje basado en juegos, entre otros; además, de los términos asociados a método, metodología y marco de referencia. Por medio de la revisión se busca responder a las preguntas: ¿Cuántas metodologías existen para el diseño de juegos serios o juegos para educar? ¿Qué características tienen en común dichas metodologías? ¿Es posible integrar dichas características en una metodología para el diseño de juegos?

Para el refinamiento de la búsqueda y selección de documentos se aplicaron un conjunto de criterios que permiten identificar las publicaciones que corresponden con mayor precisión a las preguntas de investigación planteadas, los criterios de inclusión de los documentos fueron aplicados de la siguiente manera: (1) Se tomaron publicaciones entre el año 2000 y junio de 2020, debido a que en dicho período los juegos dentro de contexto educativos tienen su mayor auge; (2) se tuvieron en cuenta las publicaciones tipo artículo, libro, capítulo de libro y documentos de conferencias, las cuales presentan la mayor parte de publicaciones de tipo científico, (3) idioma inglés, español o francés, se esta manera, se recuperaron un total de 1.590 publicaciones. Como criterios de exclusión se definieron: (1) áreas de estudio diferentes a ingeniería, ciencias de la computación, ciencias sociales y negocios o administración, debido a que son áreas de gran expansión de juegos serios, además, (2) publicaciones cuyas palabras clave no estaban directamente relacionadas con juegos, juegos serios, educación, diseño de juegos, aprendizaje basado en juegos, videojuegos, desarrollo de juegos, juegos digitales, entre otros términos afines y (3) se verificó que las publicaciones no estuvieran relacionadas con teoría de juegos, temática propia de la economía que no se encuentra dentro del objeto de estudio de la investigación. Una vez aplicados los criterios de exclusión a la búsqueda, se recuperaron un total de 522 documentos.

A partir del primer conjunto de documentos, se aprecia que la cantidad de publicaciones relacionadas con el diseño de juegos tiene una tendencia creciente, la cual toma mayor relevancia a partir del año 2010 aproximadamente y cuyo pico máximo de publicaciones se produjo en el año 2019. En la figura 1 se muestra la evolución de la cantidad de publicaciones por año en el período $2002-2020$, para el 2000 y 2001 no se encontraron publicaciones que cumplieran los criterios anteriormente expuestos.

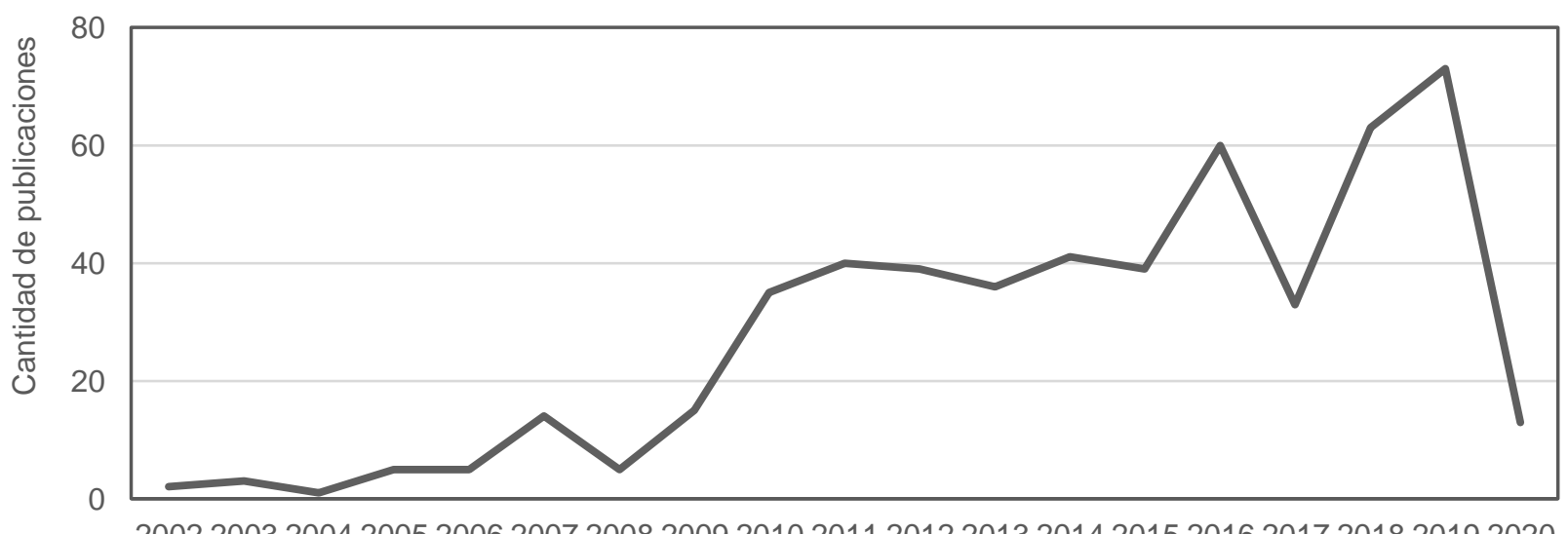

2002200320042005200620072008200920102011201220132014201520162017201820192020

Año

Fig. 1: Publicaciones relacionadas con metodologías para el diseño de juegos por año (Datos de Scopus, 2020). 
Se realizó una clasificación de los documentos teniendo en cuenta la variable "cantidad de citas", para lo cual se utilizó el indicador "Cited by $x$ documents" de Scopus, posteriormente, se evaluaron los documentos con al menos 15 citas, lo que permitió identificar 67 publicaciones que se ajustan a los criterios descritos. El principal criterio de inclusión utilizado fue documentos que presentaran metodologías generales, novedosas y propias para el diseño de juegos serios o juegos relacionados con procesos educativos, con amplia ilustración de su propuesta, con el cual se evaluó cada uno de los documentos previamente seleccionados, obteniendo un total de 17 documentos. Para complementar la revisión, se incluyeron documentos referentes teóricos que se encontraban en la base de datos Google Scholar, se aplicaron los criterios antes mencionados y se tomaron documentos con propuestas de diseño novedosas. Para el análisis de los documentos se estudió en detalle cada una de las metodologías propuestas para el diseño de juegos serios o juegos educativos y se tomaron los elementos que las componen, posteriormente, se realizó un análisis comparativo entre metodologías, en el cual se identificaron elementos comunes que serán el insumo principal para la construcción de la metodología propuesta.

\section{RESULTADOS Y DISCUSIÓN}

En total se analizaron veinticuatro (24) documentos, que incluyen doce (12) artículos en revistas, seis (6) documentos de conferencias, cuatro (4) libros y dos (2) capítulos de libro. En la tabla 1. Se muestra el detalle de los documentos examinados, se muestran autores y año de la publicación, título y una breve descripción de cada metodología propuesta.

Tabla 1: Análisis de metodologías propuestas para diseño de juegos serios.

\begin{tabular}{|c|c|c|c|}
\hline Autores y año & Documento & Descripción & Elementos de las propuestas \\
\hline Amory (2007) & $\begin{array}{l}\text { Game object model version } \\
\text { II: A theoretical framework } \\
\text { for educational game } \\
\text { development }\end{array}$ & $\begin{array}{l}\text { La propuesta describe una relación entre } \\
\text { las dimensiones pedagógicas del } \\
\text { aprendizaje y los elementos del juego. }\end{array}$ & $\begin{array}{l}\text { Definición - Aprendizaje auténtico - } \\
\text { Narrativa - Género - Colaboración social. }\end{array}$ \\
\hline $\begin{array}{l}\text { Nadolski et al. } \\
\text { (2008) }\end{array}$ & $\begin{array}{l}\text { EMERGO: A methodology } \\
\text { and toolkit for developing } \\
\text { serious games in higher } \\
\text { education }\end{array}$ & $\begin{array}{l}\text { Metodología de cinco fases y caja de } \\
\text { herramientas para el diseño de juegos } \\
\text { serios. }\end{array}$ & $\begin{array}{l}\text { Idea / Análisis - Escenario / Diseño - } \\
\text { Desarrollo - Entrega / Implementación - } \\
\text { Evaluación. }\end{array}$ \\
\hline Schell (2008) & $\begin{array}{l}\text { The art of game design: A } \\
\text { book of lenses }\end{array}$ & $\begin{array}{l}\text { Describe ampliamente aspectos del diseño } \\
\text { de juegos y propone un proceso formal, } \\
\text { también llamado bucle formal que consta } \\
\text { de siete pasos. }\end{array}$ & $\begin{array}{l}\text { Planteamiento del problema - Posibles } \\
\text { soluciones - Selección de la solución - Lista } \\
\text { de riesgos - Construir prototipos - Probar } \\
\text { prototipos - Nuevos problemas. }\end{array}$ \\
\hline $\begin{array}{l}\text { Song Y Zhang } \\
(2008)\end{array}$ & $\begin{array}{l}\text { EFM: A model for } \\
\text { educational game design. }\end{array}$ & $\begin{array}{l}\text { Modelo que describe la conexión interna } \\
\text { entre motivación, flujo, ambiente de } \\
\text { aprendizaje efectivo y juego educativo. }\end{array}$ & $\begin{array}{l}\text { Ambiente efectivo de aprendizaje - Juego } \\
\text { educativo - Experiencia de flujo - } \\
\text { Motivación. }\end{array}$ \\
\hline $\begin{array}{l}\text { Westera et al. } \\
(2008)\end{array}$ & $\begin{array}{l}\text { Serious games for higher } \\
\text { education: A framework for } \\
\text { reducing design complexity }\end{array}$ & $\begin{array}{l}\text { Se centra en reducir la complejidad de } \\
\text { diseño de juegos serios, en el nivel } \\
\text { conceptual, técnico y práctico. }\end{array}$ & $\begin{array}{l}\text { Nivel conceptual - Configuración - } \\
\text { Dinámicas - Nivel técnico - Arquitectura - } \\
\text { Nivel práctico - Estructura, } \\
\text { retroalimentación. }\end{array}$ \\
\hline $\begin{array}{l}\text { Hirumi y } \\
\text { Stapleton (2009) }\end{array}$ & $\begin{array}{l}\text { Applying Pedagogy during } \\
\text { Game Development to } \\
\text { Enhance Game-Based } \\
\text { Learning }\end{array}$ & $\begin{array}{l}\text { Muestra un proceso sistemático para el } \\
\text { diseño de juegos serios que integra } \\
\text { sistemas de diseño instruccional, con un } \\
\text { proceso de desarrollo de juegos, que } \\
\text { optimiza el aprendizaje basado en juegos. }\end{array}$ & $\begin{array}{l}\text { Desarrollo de concepto: Objetivos - } \\
\text { Aprendices, contexto. Pre-producción: } \\
\text { Métodos - Medios - Estrategia - Diseño. } \\
\text { Prototipo y producción: Materiales, } \\
\text { desarrollo - Prototipos - Evaluar - Versión. }\end{array}$ \\
\hline $\begin{array}{l}\text { Ibrahim y Jaafar } \\
(2009)\end{array}$ & $\begin{array}{l}\text { Educational games (EG) } \\
\text { design framework: } \\
\text { Combination of game } \\
\text { design, pedagogy and } \\
\text { content modeling }\end{array}$ & $\begin{array}{l}\text { Modelo de diseño de juegos educativos } \\
\text { que combina tres factores: diseño de } \\
\text { juegos, pedagogía y modelado de } \\
\text { contenido de aprendizaje. }\end{array}$ & $\begin{array}{l}\text { Diseño - Satisfacción/Eficiencia - } \\
\text { Diversión/Objetivos/Retos - Pedagogía - } \\
\text { Resultados/ Motivación - Autoaprendizaje / } \\
\text { Problemas - Modelado. }\end{array}$ \\
\hline $\begin{array}{l}\text { Yusoff et al. } \\
(2009)\end{array}$ & $\begin{array}{l}\text { A conceptual framework for } \\
\text { serious games }\end{array}$ & $\begin{array}{l}\text { El modelo propuesto incluye teoría del } \\
\text { aprendizaje y la pedagogía, combinadas } \\
\text { con requisitos de juego. }\end{array}$ & $\begin{array}{l}\text { Capacidades - Contenido instruccional - } \\
\text { Objetivos - Atributos- Actividades - } \\
\text { Reflexión - Género, mecánicas y logros. }\end{array}$ \\
\hline $\begin{array}{l}\text { Aleven et al. } \\
(2010)\end{array}$ & $\begin{array}{l}\text { Toward a framework for the } \\
\text { analysis and design of } \\
\text { educational games }\end{array}$ & $\begin{array}{l}\text { Propuesta de tres componentes principales } \\
\text { y una estrategia para combinarlos. }\end{array}$ & $\begin{array}{l}\text { Objetivos de aprendizaje - MDA: Mecánicas, } \\
\text { dinámicas, estética - Principios } \\
\text { instruccionales. }\end{array}$ \\
\hline Annetta (2010) & $\begin{array}{l}\text { The "l's" Have It: A } \\
\text { Framework for Serious } \\
\text { Educational Game Design }\end{array}$ & $\begin{array}{l}\text { Modelo de seis elementos jerárquicos para } \\
\text { diseño de juegos educativos; El elemento } \\
\text { básico: es la "identidad". }\end{array}$ & $\begin{array}{l}\text { Identidad - Inmersión - Interactividad - } \\
\text { Complejidad incremental - Enseñanza } \\
\text { informada - Diseño instruccional. }\end{array}$ \\
\hline $\begin{array}{l}\text { Brown y Wyatt } \\
(2010)\end{array}$ & $\begin{array}{l}\text { Design Thinking for Social } \\
\text { Innovation }\end{array}$ & $\begin{array}{l}\text { Cinco etapas para diseño de productos y } \\
\text { servicios; también usado en diseño de } \\
\text { juegos serios. }\end{array}$ & $\begin{array}{l}\text { Conocer a las personas - Definir el } \\
\text { problema - Idear - Prototipar - Probar. }\end{array}$ \\
\hline
\end{tabular}


Tabla 1 (continuación)

\begin{tabular}{|c|c|c|c|}
\hline Autores y año & Documento & Descripción & Elementos de las propuestas \\
\hline $\begin{array}{l}\text { Echeverría et al. } \\
\text { (2011) }\end{array}$ & $\begin{array}{l}\text { A framework for the design } \\
\text { and integration of } \\
\text { collaborative classroom } \\
\text { games }\end{array}$ & $\begin{array}{l}\text { El modelo incorpora dos dimensiones: } \\
\text { educativa (Objetivos e integración } \\
\text { pedagógica) y lúdica (Elementos de juego). }\end{array}$ & $\begin{array}{l}\text { Objetivos - Integración y modelo } \\
\text { pedagógico - Soporte tecnológico - } \\
\text { Elementos de juego - Mecánicas, historia, } \\
\text { tecnología y estética. }\end{array}$ \\
\hline Gómez (2012) & $\begin{array}{l}\text { Método para el diseño de } \\
\text { juegos orientados al } \\
\text { desarrollo de habilidades } \\
\text { gerenciales }\end{array}$ & $\begin{array}{l}\text { Metodología que consta de diez pasos } \\
\text { secuenciales para el diseño de juegos con } \\
\text { propósito educativo. }\end{array}$ & $\begin{array}{l}\text { Temática - Propósito - Objetivos - } \\
\text { Conceptos - Técnicas candidatas - } \\
\text { Selección - Incorporar diseño -Pilotos - } \\
\text { Versión final - Evaluación. }\end{array}$ \\
\hline $\begin{array}{l}\text { Mitgutsch y } \\
\text { Alvarado (2012) }\end{array}$ & $\begin{array}{l}\text { Purposeful by design?: a } \\
\text { serious game design } \\
\text { assessment framework }\end{array}$ & $\begin{array}{l}\text { La propuesta tiene seis componentes; y el } \\
\text { propósito del juego se refleja en todos los } \\
\text { elementos que soportan el diseño. }\end{array}$ & $\begin{array}{l}\text { Propósito del juego - Framing / Público } \\
\text { objetivo - Contenido / Información - } \\
\text { Mecánicas - Ficción y narrativa - Estética y } \\
\text { gráficas. }\end{array}$ \\
\hline Rooney (2012) & $\begin{array}{l}\text { A theoretical framework for } \\
\text { serious game design: } \\
\text { Exploring pedagogy, play } \\
\text { and fidelity and their } \\
\text { implications for the design } \\
\text { process }\end{array}$ & $\begin{array}{l}\text { Propone un marco teórico triádrico para el } \\
\text { diseño de juegos serios, que incluye juego, } \\
\text { pedagogía y fidelidad. }\end{array}$ & Pedagogía - Juego - Fidelidad. \\
\hline $\begin{array}{l}\text { Vanden Abeele } \\
\text { et al. (2012) }\end{array}$ & $\begin{array}{l}\text { P-III: A player-centered, } \\
\text { iterative, interdisciplinary } \\
\text { and integrated framework } \\
\text { for serious game design } \\
\text { and development. }\end{array}$ & $\begin{array}{l}\text { Metodología caracterizada por cuatro } \\
\text { pilares conceptuales: Diseño centrado en el } \\
\text { jugador, desarrollo iterativo, equipo } \\
\text { interdisciplinar e integración de juego y } \\
\text { aprendizaje. }\end{array}$ & $\begin{array}{l}\text { Concepto - Análisis de usuarios - Diseño } \\
\text { participativo - Definición del concepto - } \\
\text { Historia - Prototipos y pruebas de papel - } \\
\text { Documentar - Arte y diseño de software - } \\
\text { Prototipos y pruebas. }\end{array}$ \\
\hline $\begin{array}{l}\text { Werbach y } \\
\text { Hunter (2012) }\end{array}$ & $\begin{array}{l}\text { For the win: How game } \\
\text { thinking can revolutionize } \\
\text { your business }\end{array}$ & $\begin{array}{l}\text { La metodología llamada } 6 \mathrm{D} \text {, consta de seis } \\
\text { pasos producto de la observación de juegos } \\
\text { cotidianos y su réplica en otros contextos. }\end{array}$ & $\begin{array}{l}\text { Definir objetivos - Comportamientos } \\
\text { buscados - Describir los jugadores - Idear } \\
\text { ciclos de actividades - Diversión - } \\
\text { Implementar las herramientas. }\end{array}$ \\
\hline $\begin{array}{l}\text { Barbosa et al. } \\
(2014)\end{array}$ & $\begin{array}{l}\text { A new methodology of } \\
\text { design and development of } \\
\text { serious games }\end{array}$ & $\begin{array}{l}\text { Metodología basada en el diseño de juego } \\
\text { con misiones y conjunto de mecanismos de } \\
\text { aprendizaje. }\end{array}$ & Niveles - Mecanismos de diseño. \\
\hline $\begin{array}{l}\text { Žavcer, Mayr y } \\
\text { Petta (2014) }\end{array}$ & $\begin{array}{l}\text { Design pattern canvas: } \\
\text { Towards co-creation of } \\
\text { unified serious game } \\
\text { design patterns }\end{array}$ & $\begin{array}{l}\text { Propone una plantilla para diseñar o } \\
\text { documentar juegos existentes. Es una } \\
\text { gráfico basado en el modelo Canvas }\end{array}$ & $\begin{array}{l}\text { Investigaciones - Patrones- Datos claves - } \\
\text { Propósito - Mecánicas - Medios - Usuarios } \\
\text { - Ética - Resultados esperados. }\end{array}$ \\
\hline $\begin{array}{l}\text { Prieto, López et } \\
\text { al. (2017) }\end{array}$ & $\begin{array}{l}\text { Design methodology for } \\
\text { educational games based } \\
\text { on graphical notations: } \\
\text { Designing Urano }\end{array}$ & $\begin{array}{l}\text { La metodología comprende pasos iterativos } \\
\text { organizados en seis fases, que, además, } \\
\text { inician con tres fases preliminares. }\end{array}$ & $\begin{array}{l}\text { Desafíos, objetivos - Tipo juego - Historia - } \\
\text { Capítulos - Escenas - Educacional - } \\
\text { Emocional -De adaptación -Colaborativo. }\end{array}$ \\
\hline $\begin{array}{l}\text { Quintana y } \\
\text { García (2017) }\end{array}$ & Serious games for health & $\begin{array}{l}\text { Proponen un protocolo de diseño de juegos } \\
\text { serios de nueve pasos, que inicia con la } \\
\text { captación del "briefing", hasta el refinado } \\
\text { final. }\end{array}$ & $\begin{array}{l}\text { Objetivos - Métricas - Casos de estudio - } \\
\text { Segmentar- Motivadores - Combinar } \\
\text { elementos - Puntos - Prueba - Iterar e } \\
\text { implementar. }\end{array}$ \\
\hline García (2018) & $\begin{array}{l}\text { Canvas de diseño de } \\
\text { juegos aplicados y } \\
\text { gamificación }\end{array}$ & $\begin{array}{l}\text { Modelo Canvas con diez secciones } \\
\text { consecutivas que permiten realizar el } \\
\text { diseño de juegos de manera amigable. }\end{array}$ & $\begin{array}{l}\text { Briefing - Comportamiento - Habilidades - } \\
\text { User persona - Motivaciones - Concepto y } \\
\text { narrativa - Dónde - Restricciones- } \\
\text { Mecánicas - Recompensas. }\end{array}$ \\
\hline Nallar (2019) & $\begin{array}{l}\text { Game Design Canvas: Una } \\
\text { herramienta para el diseño } \\
\text { de juegos }\end{array}$ & $\begin{array}{l}\text { Modelo Canvas que permite realizar el } \\
\text { diseño de un juego de manera global y } \\
\text { analizar los ya existentes. }\end{array}$ & $\begin{array}{l}\text { Planeación - Deseos, necesidades - } \\
\text { Objetivos - Sistema de progresión - } \\
\text { Desafíos - Sistema de recompensas - } \\
\text { Narración. }\end{array}$ \\
\hline Silva (2020) & $\begin{array}{l}\text { Practical methodology for } \\
\text { the design of educational } \\
\text { serious games. }\end{array}$ & $\begin{array}{l}\text { Metodología que describe pasos para } \\
\text { definir mecanismos de aprendizaje. Inicia } \\
\text { con la elección del tema, objetivos y termina } \\
\text { con la experiencia del usuario. }\end{array}$ & $\begin{array}{l}\text { Objetivos - Público - Género del juego - } \\
\text { Historia, escenario, personajes - } \\
\text { Mecánicas - dinámicas - Experiencia, } \\
\text { resultados - Diversión - Capa }\end{array}$ \\
\hline
\end{tabular}

A partir del análisis realizado fue posible establecer que existen numerosas metodologías dirigidas al diseño de juegos serios, sin embargo, la mayor parte de las propuestas se enfocan en áreas específicas del conocimiento, es decir, son planteamientos para el diseño de un conjunto reducido de juegos serios en áreas determinadas de medicina, ingeniería, obesidad, educación preescolar, entre otros o centradas en la inclusión de elementos específicos dentro de los diseños, lo cual hace complejo vislumbrar y seleccionar metodologías adecuadas para el diseño de juegos serios. De esta manera, se analizó cada una de las metodologías para el diseño de juegos serios o juegos educativos, se tomaron cada uno de los elementos que conforman las propuestas y se realizó una evaluación de los mismos, para luego, compararlos y, de esta manera, encontrar 
elementos comunes que tienen dichas metodologías. A partir del análisis y mediante el método inductivo, tomando como base los criterios específicos de las propuestas analizadas, fue posible identificar y establecer criterios generales, que permiten agrupar los elementos comunes identificados en las propuestas. En la tabla 2, se muestran los criterios generales establecidos para realizar la clasificación, los elementos y términos encontrados en las metodologías analizadas y la cantidad de documentos que incluyen dicho criterio dentro de su propuesta. Los criterios generales encontrados, serán la base fundamental para la metodología de diseño de juegos serios que posteriormente se propondrá.

Tabla 2: Elementos de metodologías analizadas para diseño de juegos.

\begin{tabular}{|c|c|c|c|}
\hline$\#$ & Criterios Establecidos & Elementos de metodologías analizadas & Cantidad \\
\hline \multirow{7}{*}{1} & \multirow{7}{*}{$\begin{array}{l}\text { Establecer objetivos del } \\
\text { juego: Determinar objetivos } \\
\text { y propósitos a alcanzar por } \\
\text { medio de la propuesta. }\end{array}$} & Planteamiento del problema & \multirow{7}{*}{$\begin{array}{l}\text { Investigaciones que } \\
\text { incluyen alguna variante } \\
\text { del criterio: } \\
19 \text { / } 24-79,2 \%\end{array}$} \\
\hline & & Definir objetivos & \\
\hline & & Propósito del juego & \\
\hline & & Planeación & \\
\hline & & Idea a desarrollar & \\
\hline & & Comportamientos deseados & \\
\hline & & Fase de análisis & \\
\hline \multirow{9}{*}{2} & \multirow{9}{*}{$\begin{array}{l}\text { Caracterizar al público } \\
\text { objetivo: Identificar } \\
\text { características propias de } \\
\text { usuarios a quienes se } \\
\text { dirige la propuesta. }\end{array}$} & Conocer a las personas & \multirow{9}{*}{$\begin{array}{l}\text { Investigaciones que } \\
\text { incluyen alguna variante } \\
\text { del criterio: } \\
14 \text { / } 24-58,3 \%\end{array}$} \\
\hline & & Describir a los jugadores & \\
\hline & & Segmentar a las personas & \\
\hline & & Identidad & \\
\hline & & Público objetivo & \\
\hline & & Análisis de los usuarios & \\
\hline & & Usuarios & \\
\hline & & Analizar aprendices y contexto & \\
\hline & & Motivaciones & \\
\hline \multirow{5}{*}{3} & \multirow{5}{*}{$\begin{array}{l}\text { Plantear soluciones y tipo } \\
\text { de juego: Clasificar tipo de } \\
\text { juego a proponer. }\end{array}$} & Posibles soluciones & \multirow{5}{*}{$\begin{array}{l}\text { Investigaciones que } \\
\text { incluyen alguna variante } \\
\text { del criterio: } \\
7 \text { / } 24-29,2 \%\end{array}$} \\
\hline & & Técnicas candidatas & \\
\hline & & Género del juego & \\
\hline & & Diseño del tipo de juego & \\
\hline & & Estrategias de aprendizaje & \\
\hline \multirow{7}{*}{4} & \multirow{7}{*}{$\begin{array}{l}\text { Estudio de la literatura: } \\
\text { Revisar literatura de } \\
\text { temática a tratar o de } \\
\text { juegos previos } \\
\text { relacionados. }\end{array}$} & Definición de conceptos & \multirow{7}{*}{$\begin{array}{l}\text { Investigaciones que } \\
\text { incluyen alguna variante } \\
\text { del criterio: } \\
12 \text { / } 24-50,0 \%\end{array}$} \\
\hline & & Mostrar casos de estudio & \\
\hline & & Contenido / Información & \\
\hline & & Investigaciones relacionadas & \\
\hline & & Nivel conceptual & \\
\hline & & Integración pedagógica & \\
\hline & & Aprendizaje auténtico & \\
\hline \multirow{18}{*}{5} & \multirow{18}{*}{$\begin{array}{l}\text { Incorporar elementos de } \\
\text { juego: Definir y describir } \\
\text { detalladamente los } \\
\text { elementos que componen } \\
\text { el juego diseñado y el } \\
\text { funcionamiento. }\end{array}$} & $\begin{array}{l}\text { Incorporar diseño y conocimiento al } \\
\text { juego }\end{array}$ & \multirow{18}{*}{$\begin{array}{l}\text { Investigaciones que } \\
\text { incluyen alguna variante } \\
\text { del criterio: } \\
24 \text { / } 24-100 \%\end{array}$} \\
\hline & & Idear ciclos de actividades & \\
\hline & & Diseño de capítulos, escenas. & \\
\hline & & Desafíos & \\
\hline & & Definir motivadores & \\
\hline & & Combinar elementos de juego & \\
\hline & & Diversión & \\
\hline & & Sistemas de puntuación / Recompensas & \\
\hline & & Diseño instruccional & \\
\hline & & Seleccionar técnicas & \\
\hline & & Narrativa / Historia / Personajes & \\
\hline & & Mecánicas / Componentes & \\
\hline & & Dinámicas & \\
\hline & & Estética / Diseño & \\
\hline & & Tecnología / Medios & \\
\hline & & Soporte tecnológico & \\
\hline & & Sistema de progresión & \\
\hline & & Atributos del juego & \\
\hline \multirow{8}{*}{6} & \multirow{8}{*}{$\begin{array}{l}\text { Realizar prototipo / Prueba } \\
\text { del diseño o prototipo: } \\
\text { Ejecutar y aplicar versiones } \\
\text { preliminares del juego } \\
\text { propuesto. }\end{array}$} & Construir / Generar prototipos & \multirow{8}{*}{$\begin{array}{l}\text { Investigaciones que } \\
\text { incluyen alguna variante } \\
\text { del criterio: } \\
12 / 24-50 \%\end{array}$} \\
\hline & & Prototipos y pruebas & \\
\hline & & Probar prototipos & \\
\hline & & Sesiones piloto & \\
\hline & & Implementar las herramientas & \\
\hline & & Fase de prueba & \\
\hline & & Entrega / Implementación & \\
\hline & & Nivel práctico & \\
\hline
\end{tabular}


Tabla 2 (continuación)

\begin{tabular}{|c|c|c|c|}
\hline \# & Criterios Establecidos & Elementos de metodologías analizadas & Cantidad \\
\hline \multirow{9}{*}{7} & \multirow{9}{*}{$\begin{array}{l}\text { Refinar / Evaluar el juego: } \\
\text { Aplicar continuamente } \\
\text { prototipos y obtener } \\
\text { mejoras, evaluación y } \\
\text { perfeccionamiento de la } \\
\text { propuesta. }\end{array}$} & Agregar capaz de aprendizaje & \multirow{9}{*}{$\begin{array}{l}\text { Investigaciones que } \\
\text { incluyen alguna variante } \\
\text { del criterio: } \\
9 / 24-37,5 \%\end{array}$} \\
\hline & & Iterar e implementar & \\
\hline & & Diseño colaborativo & \\
\hline & & Prototipo final & \\
\hline & & Resultados esperados & \\
\hline & & Versión final del juego & \\
\hline & & Retroalimentación / Estructura & \\
\hline & & Implementación y evaluación & \\
\hline & & Evaluación & \\
\hline \multirow{7}{*}{8} & \multirow{7}{*}{$\begin{array}{l}\text { Otros elementos } \\
\text { particulares: Elementos } \\
\text { adicionales que no } \\
\text { pudieron ser clasificados en } \\
\text { categorías anteriores. }\end{array}$} & Lista de riesgos & \multirow{7}{*}{$\begin{array}{l}\text { Investigaciones que } \\
\text { incluyen alguna variante } \\
\text { del criterio: } \\
8 / 24-33,3 \%\end{array}$} \\
\hline & & Encuesta de evaluación & \\
\hline & & Reflexión & \\
\hline & & Fidelidad & \\
\hline & & Ética & \\
\hline & & Datos claves & \\
\hline & & Medio / Plataforma & \\
\hline
\end{tabular}

Se establecieron un total de ocho criterios generales que abarcan los elementos analizados en las metodologías estudiadas. Es importante mencionar que el criterio (5) incorporar elementos de juego, está presente en el $100 \%$ de los documentos evaluados, además, los criterios: (1) Establecer objetivos de juego, (2) caracterizar el público objetivo, (4) estudio de la literatura y (6) realizar prototipo / prueba del diseño o prototipo, se encontraron en por lo menos el $50 \%$ de los documentos; Por otro lado, el criterio (3) plantear soluciones y tipo de juego no se incluyó debido a que no existe una clasificación general de juegos que permita categorizar todas las propuestas, al igual que el criterio (8) otros elementos, ya que correspondían a particularidades de las propuestas analizadas; los demás criterios fueron incluidos en la propuesta. De esta manera, a partir de los criterios generales establecidos, se propone una metodología para el diseño de juegos serios, tanto para aplicaciones físicas como para ambientes virtuales, la cual muestra un proceso secuencial e integrativo, ya que las propuestas analizadas no realizan un análisis comparativo-integrativo, que permitirá al diseñador incluir los elementos necesarios en el juego y a su vez, realizar una evaluación de funcionalidad por medio de la iteración. La metodología propuesta consta de tres fases, tomando como base la propuesta de Hirumi y Stapleton (2009) y complementadas con los hallazgos anteriores: (1) fase de pre-diseño, también llamada fase de planeación, (2) fase de diseño, con dos sub-fases: escrita y de construcción, y (3) fase de testeo, cada una de las cuales posee elementos específicos y pasos a seguir para el diseño del instrumento requerido. En la figura 2, se muestra el modelo metodológico integrador propuesto.

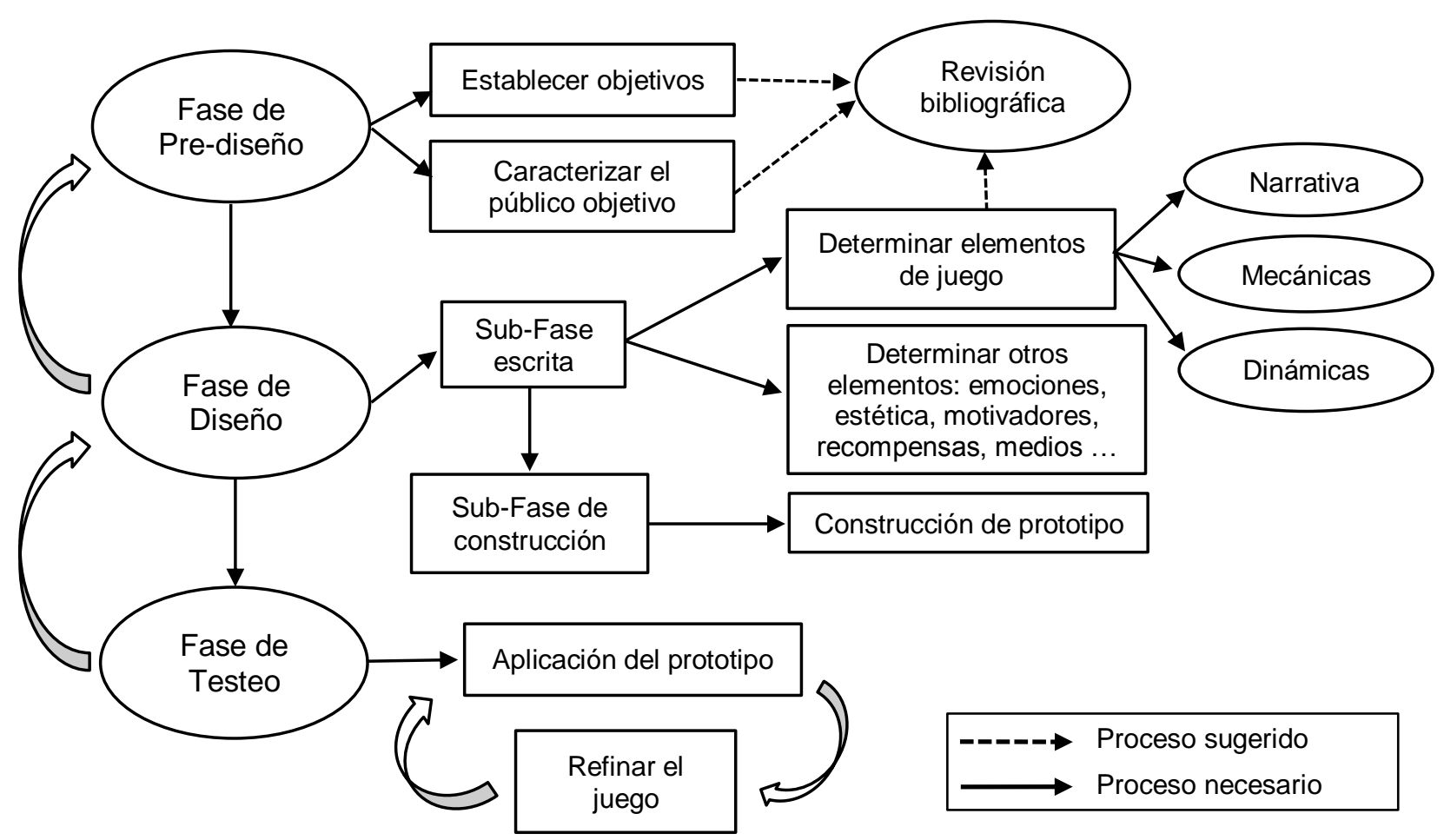

Fig. 2: Modelo metodológico integrador para el diseño de juegos serios. 


\section{Fase de pre-diseño}

La primera fase de la metodología de diseño propuesta, es la fase de prediseño, en la cual se realiza la planeación del juego que se quiere diseñar. Dentro de esta fase, primero es necesario establecer los objetivos a alcanzar por medio de la aplicación de la herramienta, es decir, lo que se quiere lograr con esta; entre los más relevantes se encuentran enseñar conceptos, medir características, reforzar conocimientos existentes, entrenar y evaluar; lo anterior se consideró necesario dado que la propuesta se centra en el diseño desde los elementos del juego y no se hace énfasis en el proceso de desarrollo, por lo cual establecer y tener claros los objetivos, permitirá enfocar acertadamente las fases siguientes e incluir elementos que permitan su cumplimiento. Posteriormente, se debe definir de manera precisa la población a la cual se aplicará la herramienta y es necesario realizar una caracterización de la misma, con el fin de identificar motivaciones, gustos, pasatiempos, actividades, entre otras, que puedan ser incorporadas en el diseño del juego y que a su vez lo haga más atractivo para los participantes. Durante la primera fase, es recomendable realizar una revisión de literatura en dos sentidos: (1) de conceptos teóricos que se quieren incorporar en el juego, que permita tener clara la temática y otorgue variables que se puedan integrar al diseño de la herramienta, y (2) de juegos propuestos por otros autores que traten una temática igual o similar, esto con el objetivo de determinar cómo otros autores abordan la temática o conceptos y qué elementos funcionan de manera correcta y cuáles no lo hacen. Dicha revisión es sugerida más no necesaria, debido a que dependerá de la experticia de los diseñadores, del conocimiento que tengan sobre la temática a incluir en el juego y de la profundidad con que se desee abordar.

\section{Fase de diseño}

Una vez se tenga lista la primera fase, se inicia la fase de diseño, en la cual se determinan los diferentes elementos que contendrá la herramienta propuesta. Ésta fase cuenta con dos sub-etapas que se realizan de manera secuencial: (1) sub-etapa escrita y (2) sub-etapa de construcción, cada una de las cuales tiene a su vez algunos elementos a determinar. Ésta etapa está relacionada con la revisión de literatura, debido a que en ella se integran los elementos o variables resultantes de la revisión. En la etapa escrita, se documenta cada una de las ideas y elementos que se vayan a integrar al juego. Inicialmente se deben determinar (1) narrativa, historia o contexto en que se enmarca el juego, (2) mecánicas, elementos núcleo que integran el juego, los componentes particulares, y (3) dinámicas, que describen cómo los participantes seguirán las mecánicas del juego. Algunos de los principales elementos de juego que integran dichas categorías son: Personajes, temática, espacios, atributos, reglas, sistema de recompensas o de puntuación, castigos, nivel de dificultad restricciones, manera en que el juego evolucionará o etapas que tendrá, emociones de los participantes, estética o arte del juego, motivadores intrínsecos y extrínsecos a integrar, medios, materiales o tecnología a utilizar, entre otros elementos que se determinan según la necesidad del diseñador y de los objetivos que se quieren lograr.

Al finalizar la etapa escrita y tener definidos los elementos que conformarán el juego propuesto, se inicia la etapa de construcción, en la cual se realiza un prototipo del juego planteado y de todos los elementos que se incluyeron en él, de la manera más fiel y detallada posible. Es en esta etapa donde se pasa a la acción y construcción física o virtual del juego planteado anteriormente.

\section{Fase de testeo}

La fase final del proceso de diseño es la fase de testeo, en la cual el prototipo construido debe ser aplicado a un grupo pequeño de personas que representan la población objetivo, esto con el fin de observar el comportamiento de los participantes, funcionamiento del juego e identificar posibles oportunidades de mejora, pero también preguntando a los participantes percepciones, experiencias, opiniones y propuestas para el juego. Es una fase iterativa en la cual cada vez que se aplica el prototipo, se ajustan los elementos necesarios para refinar el juego y se vuelve a aplicar, con el objetivo de perfeccionar su funcionamiento. Adicionalmente, es posible que en la fase de testeo se detecten errores, imprecisiones o falta de consistencia entre algunos elementos integrados en las fases de pre-diseño o diseño, por lo cual es factible y necesario volver a dichas fases las veces que sea necesario, para corregir y mejorar los detalles identificados, sin embargo, es necesario tener en cuenta que una vez se regrese en el proceso de diseño, es necesario volver a ejecutar y verificar todas las fases y elementos de los pasos posteriores. Para determinar el fin de la fase de testeo y, la puesta a punto del juego serio propuesto, es necesario que el diseñador observe cuidadosamente que todos los elementos integrados al juego funcionen correctamente y de la forma deseada, de igual manera, los participantes de las pruebas brindan información importante y expresan la pertinencia de realizar modificaciones adicionales al diseño.

Además del proceso formal y estructurado de diseño de juegos serios, es necesario tener en cuenta otros elementos que aportan al exitoso diseño de las propuestas: (1) segmentación de la población objetivo, dado 
que las dos variables demográficas más importantes para los diseñadores de juegos son edad y género (Schell, 2008), (2) tipos de jugadores, no todas las personas juegan de la misma manera, ni tienen los mismos objetivos (Bartle, 1996) y (3) balancear (Balancing) los elementos del juego, es decir, equilibrar y comprender las relaciones que se establecen entre dichos elementos de diseño, de manera que se manipulen correctamente para obtener la experiencia deseada (Schell, 2008) siempre teniendo presentes los objetivos educativos. La segmentación de la población hace parte de la fase de pre-diseño del modelo propuesto, específicamente en la caracterización del público objetivo, sin embargo, se incluye dentro de los elementos adicionales a considerar, debido a que estos son propuestas específicas de los autores mencionados, que no constituyen un método para el diseño de juegos serios, adicionalmente, complementan el proceso y aportan al éxito de los juegos diseñados.

\section{CONCLUSIONES}

A partir de la revisión bibliográfica se evidencia que existen numerosas propuestas para el diseño de juegos serios o juegos educativos, sin embargo, la mayor parte de éstas se centran en proponer metodologías para áreas específicas del conocimiento como medicina, ingeniería, obesidad, educación preescolar, evitar ataques cibernéticos, juegos en organizaciones, entre otros o para la inclusión o creación de elementos específicos de los juegos, como la creación de escenarios, logros, inclusión de mecánicas específicas, entre otras, situación que resta claridad para los diseñadores de juegos, a la hora de definir una metodología adecuada para el diseño de las herramientas educativas.

De esta manera, por medio del análisis de modelos y metodologías para el diseño de juegos serios o juegos educativos, fue posible concluir que existen cinco criterios generales importantes a la hora de diseñar juegos serios, los cuales son: incorporar elementos de juego (100\%), establecer objetivos del juego $(79,2 \%)$, caracterizar el público objetivo $(58,3 \%)$, estudio de la literatura $(50,0 \%)$ y realizar prototipo / prueba del diseño o prototipo $(50,0 \%)$; los porcentajes indican la proporción de documentos analizados en los cuales se identificó cada uno de los criterios. Además, se identificaron otros criterios utilizados con menor frecuencia: Refinar / evaluar el juego $(37,5 \%)$, plantear soluciones y tipo de juego $(29,2 \%)$ y otros elementos particulares $(33,3 \%)$.

El modelo metodológico propuesto es una herramienta novedosa, sencilla e intuitiva, basada en criterios producto del análisis de otras metodologías y la cual puede ser utilizada en el diseño de juegos serios tanto de manera presencial, como en ambientes virtuales, siguiendo las tres fases propuestas: pre-diseño, diseño y testeo; dando al diseñador las herramientas necesarias para realizar su propia propuesta, incluso si no se tiene experiencia en el área. Realizar un diseño de juegos serios exitoso y efectivo, requiere rigurosidad en el seguimiento y desarrollo de cada una de las fases y actividades propuestas en el modelo metodológico presentado.

\section{REFERENCIAS}

Aleven, V., Myers, E., Easterday, M., y Ogan, A., Toward a Framework for the Analysis and Design of Educational Games, https://doi.org/10.1109/DIGITEL.2010.55, The 3rd IEEE International Conference on Digital Game and Intelligent Toy Enhanced Learning - DIGITEL 2010, 69-76 (2010).

Amory, A., Game Object Model Version II: A Theoretical Framework for Educational Game Development, https://doi.org/10.1007/s11423-006-9001-x, Educational Technology Research and Development, 55(1), 51-77 (2007).

Annetta, L.A., The “l's" Have It: A Framework for Serious Educational Game Design, https://doi.org/10.1037/a0018985, Review of General Psychology, 14(2), 105-112 (2010).

Barbosa, A.F.S., Pereira, P.N.M., Dias, J.A.F.F., y Silva, F.G.M., A New Methodology of Design and Development of Serious Games, https://doi.org/10.1155/2014/817167, International Journal of Computer Games Technology, 1-8 (2014).

Bartle, R., Hearts, Clubs, Diamons, Spades: Players who Suit MUDS, Journal of MUD Research, 1(1), 19 (1996).

Breuer, J., y Bente, G., Why so Serious? On the Relation of Serious Games and Learning, Eludamos Journal for Computer Game Culture, ISSN: 1866-6124, 4(1), 7-24 (2010).

Brown, T., y Wyatt, J., Design Thinking for Social Innovation, Standford Social Innovation Review, Invierno, 29-35 (2010).

Djaouti, D., Alvarez, J., Jessel, J.P., y Rampnoux, O., Origins of Serious Games, en: Serious Games and Edutainment Applications, 25 - 43. Springer, Londres, Inglaterra (2011).

Echeverría, A., García-Campo, C. , y otros cinco autores, A Framework for the Design and Integration of Collaborative Classroom Games, https://doi.org/10.1016/j.compedu.2010.12.010, Computers and Education, 57(1), 1127-1136 (2011).

García, O., Canvas de Diseño de Juegos Aplicados y Gamificación. Barcelona, España (2018).

Gómez, M.C., Método para el Diseño de Juegos Orientados al Desarrollo de Habilidades Gerenciales, en: Juegos Gerenciales, 39 - 58. Universidad Nacional de Colombia, Medellín, Colombia (2012).

Hirumi, A., y Stapleton, C., Applying Pedagogy during Game Development to Enhance Game-Based Learning, 
https://doi.org/10.1007/978-0-387-09775-6 en: Games: Purpose and Potential in Education, 127 - 162. Springer, Boston, Estados Unidos (2009).

Ibrahim, R., y Jaafar, A., Educational Games (EG) Design Framework: Combination of Game Design, Pedagogy and Content Modeling, https://doi.org/10.1109/ICEEl.2009.5254771, Proceedings of the 2009 International Conference on Electrical Engineering and Informatics - ICEEI 2009, Agosto, 293-298 (2009).

Michael, D. R., y Chen, S., Serious Games: Games that Educate, Train, and Inform, 17 - 27. Thomson Course Technology, Boston, Estados Unidos (2006).

Mitgutsch, K., y Alvarado, N., Purposeful by Design?: a Serious Game Design Assessment Framework, https://doi.org/10.1145/2282338.2282364, en: Proceedings of the International Conference on the Foundations of Digital Games (FDG '12), ACM, 121-128 (2012).

Mogrovejo, A. B., Mamani, G., y Tipo, M. L., Game and Simulation of Television Contest Programs as a Didactic Technique to Improve the Learning of English Vocabulary in Spanish-Speaking Students, https://doi.org/10.4067/S071807642019000100225, Información Tecnológica, 30(1), 225-236, (2019).

Nadolski, R.J., Hummel, H.G.K., y otros cinco autores, EMERGO: A Methodology and Toolkit for Developing Serious Games in Higher Education, https://doi.org/10.1177/1046878108319278, Simulation and Gaming, 39(3), 338-352 (2008).

Nallar, D., Game Design Canvas: Una Herramienta para el Diseño de Juegos, Game Design LA, 1 - 8 (2019).

Prieto, R., López, J.R., y otros tres autores, Design Methodology for Educational Games Based on Graphical Notations: Designing Urano, https://doi.org/10.1016/j.entcom.2016.08.005, Entertainment Computing, 18, 1-14 (2017).

Quintana, Y., y García, O., Serious Games for Health, 105 - 124. Editoria Gedisa S.A., Barcelona, España (2017).

Rooney, P., A Theoretical Framework for Serious Game Design: Exploring Pedagogy, Play and Fidelity and their Implications for the Design Process, https://doi.org/10.4018/ijgbl.2012100103, International Journal of Game-Based Learning, 2(4), 41-60 (2012).

Schell, J., The Art of Game Design: A Book of Lenses. 57 - 95. Morgan Kaufmann Publishers, Burlington, Estados Unidos (2008).

Silva, F.G.M., Practical Methodology for the Design of Educational Serious Games, https://doi.org/10.3390/info11010014, Information (Switzerland), 11(1), 1-13 (2020).

Song, M., y Zhang, S., EFM: A Model for Educational Game Design, https://doi.org/10.1007/978-3-540-69736-7_54, Lecture Notes in Computer Science (Including Subseries Lecture Notes in Artificial Intelligence and Lecture Notes in Bioinformatics), 5093 LNCS, 509-517 (2008).

Vanden Abeele, V., De Schutter, B., y otros ocho autores, P-III: A Player-Centered, Iterative, Interdisciplinary and Integrated Framework for Serious Game Design and Development, https://doi.org/10.1007/978-3-642-33814-4_14, Communications in Computer and Information Science, 280, 82-86 (2012).

Velásquez, J.D., Una Guía Corta para Escribir Revisiones Sistemáticas de Literatura parte 4 , https://doi.org/10.15446/dyna.v82n190.49511, DYNA, 82(190), 9-12 (2015).

Werbach, K., y Hunter, D., For the Win: how Game Thinking can Revolutionize your Business, 1ra edición, 28 - 43. Wharton Digital Press, Philadelphia, Estados Unidos (2012).

Westera, W., Nadolski, R.J., Hummel, H.G.K., y Wopereis, I.G.J.H., Serious Games for Higher Education: A Framework for Reducing Design Complexity, https://www.doi.org/10.1111/j.1365-2729.2008.00279.x, Journal of Computer Assisted Learning, 24(5), 420-432 (2008).

Yusoff, A., Crowder, R., Gilbert, L., y Wills, G., A Conceptual Framework for Serious Games, https://www.doi.org/10.1109/ICALT.2009.19, Proceedings - 2009 9th IEEE International Conference on Advanced Learning Technologies, ICALT 2009, 21-23 (2009).

Žavcer, G., Mayr, S., y Petta, P., Design Pattern Canvas: Towards Co-Creation of Unified Serious Game Design Patterns, https://www.doi.org/10.1109/VS-Games.2014.7012153, 6th International Conference on Games and Virtual Worlds for Serious Applications (VS-GAMES), IEEE, 1-2 (2014).

Zyda, M., From Visual Simulation to Virtual Reality to Games, Computer, ISSN: 0018-9162, 38(9), 25-32 (2005). 